\title{
The accuracy of Multi-detector row CT for the assessment of tumor invasion of the mesorectal fascia in primary rectal cancer
}

\author{
Roy Vliegen, ${ }^{1}$ Raphaela Dresen, ${ }^{1}$ Geerard Beets, ${ }^{2}$ Alette Daniels-Gooszen, ${ }^{1}$ Alfons \\ Kessels, ${ }^{3}$ Jos van Engelshoven, ${ }^{1}$ Regina Beets-Tan ${ }^{1}$ \\ ${ }^{1}$ Department of Radiology, University Hospital of Maastricht, Maastricht, The Netherlands \\ ${ }^{2}$ Department of Surgery, University Hospital of Maastricht, Maastricht, The Netherlands \\ ${ }^{3}$ Department of Statistics, University Hospital of Maastricht, Maastricht, The Netherlands
}

\begin{abstract}
Purpose: To evaluate the accuracy of Multi-detector row CT (MDCT) for the prediction of tumor invasion of the mesorectal fascia (MRF).

Materials and methods: A total of 35 patients with primary rectal cancer underwent preoperative staging magnetic resonance imaging (MRI) and MDCT. The tumor relationship to the MRF, expressed in 3 categories $(1$-tumor free $\mathrm{MRF}=$ tumor distance $\geq 1 \mathrm{~mm}$; 2 -threatened $=$ distance $<1 \mathrm{~mm} ; 3$-invasion $=$ distance $0 \mathrm{~mm}$ ) was determined on CT by two observers at patient level and at different anatomical locations. A third expert reader evaluated the MRF tumor relationship on MRI, which served as reference standard. Receiver operating characteristic curves (ROC-curves) and areas under these curves (AUC) were calculated. The inter-observer agreement of CT was determined by using linear weighted kappa statistics.

Results: The AUC of CT for MRF invasion was 0.71 for observer 1 and 0.62 for observer 2. The inter-observer agreement was kappa $=0.34$. The performance of CT at mid-high rectal levels was statistically significant better compared to low anterior (obs. 1 : $\mathrm{AUC}=0.88$ vs. 0.50 ; obs 2 : $\mathrm{AUC}=0.84$ vs. $0.31 ; P \leq 0.040)$.

Conclusion: Multi-detector row CT has a poor accuracy for predicting MRF invasion in low-anterior located tumors. The accuracy of $\mathrm{CT}$ significantly improves for tumors in the mid-high rectum. There is a high inconsistency among readers.
\end{abstract}

Correspondence to: Roy Vliegen; email: royvliegen@ hetnet.nlrvn05@atriummc.nl
Key words: Magnetic resonance imaging-Computed tomography—Staging-Rectal-Cancer

The prognosis of patients with rectal cancer has improved since the introduction of total mesorectal excision (TME) surgery [1-3]. Using this surgical technique the mesorectal compartment including the rectum and perirectal fat is completely excised by sharp dissection along the mesorectal fascia (MRF) [1]. Additionally, large randomized trials have shown that neo-adjuvant therapy improves local tumor control even further, regardless of optimized surgical techniques [3, 4]. The advances in rectal cancer treatment have provoked differentiated neo-adjuvant treatment strategies based on anatomical preoperative identifiable risk factors for local tumor recurrence as can be visualized with magnetic resonance imaging (MRI) [5]. One of the most important risk factors is the tumor relationship to the MRF, which actually defines the surgical circumferential resection margin (CRM) in TME surgery [6, 7]. Long courses of neoadjuvant chemoradiation have emerged as the preferential treatment of patients with anticipated tumor invasion of the MRF on MRI in order to downstage/downsize the tumor and to obtain tumor free resection margins [5].

Magnetic resonance imaging has become an integral part of the diagnostic work-up of patients with rectal cancer due to its proven efficacy to determine the tumor relationship to the MRF [5, 8-11]. However, the moderate availability [12] and the higher cost of MR on one hand, and improved Multi-detector row CT (MDCT) on the other hand, have revived the discussion whether to 
use CT or MRI for rectal cancer staging. With these state of the art CT technique it has become feasible to visualize the MRF and to perform a quick one stop shop examination of the whole abdomen including distant metastasis. At present, only a few MDCT studies have been published on the subject of rectal cancer staging [13-17]. To our knowledge, two studies focused on the prediction of tumor invasion of the MRF [13,18]. One study with MDCT suggested results equal to MRI, and the other with conventional CT suggested results inferior to MRI. The question on the accuracy of MDCT remains open.

The application of chemoradiation as the prefered treatment of locally advanced rectal cancer has created a major methodological problem for the evaluation of the staging accuracy of new imaging techniques. Due to the tumor downsizing the traditional gold standard of histology is no longer valid. Subgroup analysis of patients treated with short-courses of $5 \times 5$ Gy radiotherapy (no downstaging or downsizing effects) or without neoadjuvant therapy obviously introduces a selection bias of small tumors in which imaging of tumor invasion of the MRF cannot be assessed because there is no invasion.

Therefore, the aim of the present study was to evaluate the accuracy of MDCT for the prediction of tumor invasion of the MRF with MRI as reference standard.

\section{Materials and methods}

\section{Patients}

Institutional approval was obtained for this retrospective study. The records of a cohort of consecutive patients with biopsy proven primary rectal cancer were searched for patients who underwent both MRI and MDCT imaging at the University Hospital Maastricht between June 2004 and June 2006. The standard workup for patients with a rectal cancer includes a pelvic MRI. Some patients received a MDCT because of preoperative planning of radiotherapy. Inclusion criteria were availability of both MRI and contrast enhanced CT examinations of good quality prior to the application of any neo-adjuvant therapy. In total, 35 patients met these inclusion criteria and were enrolled in the present study. Relevant clinical information such as type of neo-adjuvant therapy, type of operation, radiologic and histologic reports were recorded. There were 23 men and 12 women. The mean age of the patients was 68 years (range 44-85 years). The mean time between the MRI and CT examination was 24 days (range 1-49 days).

\section{Technique}

All CT studies were performed on a 16-slice CT system (SOMATOM $^{\circledR}$ Sensation 16, Siemens, Erlangen, Germany). The scan protocol comprised a slice collimation of $16 \times 0.75 \mathrm{~mm}$, table feed/rotation: $15.0 \mathrm{~mm}$, rotation time $0.5 \mathrm{~s}$, reconstruction increment $5 \mathrm{~mm}, 5 \mathrm{~mm}$ reconstructed slice thickness. All the patients received $120 \mathrm{ml}$ of nonionic IV contrast agent (Iobitridol $350 \mathrm{mg}$, Xenetix ${ }^{\circledR}$, Guerbet, France) at a flow rate of $3 \mathrm{ml} / \mathrm{sec}$ and oral contrast (Telebrix gastro ${ }^{\circledR}$, Guerbet). The images of the pelvis were obtained in the porto-venous contrast phase (scan delay $60 \mathrm{~s}$ ). None of the patients received a contrast enema or bowel relaxation.

Magnetic resonance (MR) imaging was performed on a 1.5 Tesla system (Gyroscan, Powertrack 6000 NT, Philips Medical Systems, The Netherlands) using a phased array sense-cardiac coil. The scan protocol consisted of a T2-weighted sequence obtained in three orientations (axial, sagittal and coronal): turbo(fast)spin echo, $\mathrm{TR}=3427 / \mathrm{TE}=150 \mathrm{msec}$, echo train length of 25, $4 \mathrm{~mm}$ slice thickness, $0.8 \mathrm{~mm}$ gap, 6 signal averages, $175 \times 256$ matrix, $20 \mathrm{~cm}$ FOV. None of the patients received Gadolinium contrast enhanced sequences, fat suppression tecniques, rectal contrast enema or bowel relaxation.

\section{Image evaluation}

The CT examinations were read by two observers who were blinded to each other and to the clinical and MRI results. Observer 1 was a dedicated abdominal radiologist with over 8 years of experience in reading pelvic CT and MRI (R.V.). Observer 2 was a general radiologist with over 5 years of experience in reading cross sectional imaging (A.D.G). The MRI examinations were assessed by a third observer with over 12 years experience in rectal cancer MRI and who has read more than 1500 MRIs of rectal cancer cases (R.B.T).

Observers 1 and 2 assessed the relationship between the rectal tumor and the MRF on the CT images, and the third observer on MRI. The MRF was defined as the fine linear structure surrounding the mesorectal compartment, hypo-intense on T2-weighted MR images and isointense to muscle on CT. The relation of the rectal tumor to the MRF was expressed in 3 discrete categories: $1=$ tumor free MRF defined as a measured distance between the tumor periphery and the MRF of $\geq 1 \mathrm{~mm}$; $2=$ tumor threatened MRF defined as a distance of $<$ $1 \mathrm{~mm} ; 3=$ tumor invaded MRF defined as a distance of $=0 \mathrm{~mm}$ between the tumor and the MRF $[9,11]$. All image evaluations and measurements were performed on a viewing station.

Specific anatomical locations of the MRF were individually evaluated for the presence of rectal cancer and, if present, the tumor relation to the MRF was assessed according to the previously described criteria 1-3. Anatomical locations were defined as:

1 Low anterior (anterior mesorectal quadrant at the level of the prostate and vagina).

2 Low lateral left, posterior and lateral right (lateral and posterior mesorectal quadrants at the level of the 
pelvic floor upto the level of the obturator muscles and low sacrum).

3 Mid anterior in males (anterior mesorectal quadrant at the level of the seminal vesicles).

4 Mid-high lateral left, posterior and lateral right (lateral and posterior mesorectal quadrants at the level of the obturator muscles upto the piriform muscles and hig sacrum).

\section{Statistical analysis}

The CT findings at patient level and at the level of different anatomical locations were compared with the corresponding MRI findings, serving as the reference standard. Receiver operating characteristic curves (ROC curves) were constructed and areas under the curves (AUC) were calculated by using the scored categories 13. The difference in performance of MDCT for the prediction of MRF invasion for different anatomical locations was analysed by means of comparison of the corresponding AUC and the calculation of a critical ratio $\mathrm{z}$ according to the method of Hanley [19]. For this analysis, only anatomical locations with abnormal rectal walls on MRI were included [19]. A $P$ value of $<0.05$ was considered as statistically significant. Accuracy, sensitivity, specificity, positive predictive value (PPV), and negative predictive value (NPV) of CT for the prediction of tumor invasion of the MRF was calculated for both observers by dichotomization of the scored discrete categories $1-3$ (cutoff between category 1 vs. $2-3$ ). The inter-observer agreement for CT was determined by using linear weighted kappa statistics based on the scored discrete categories 1-3 [20]. Statistical analysis was performed by using the software package SPSS for Windows $^{\circledR}$, release 11.5 (Chicago, IL, USA).

\section{Results}

\section{Reference standard MRI vs. clinical results}

In total, 15 of 35 patients had locally advanced rectal tumors with tumor invasion of the MRF on MRI. All of these 15 patients were treated with long courses of chemoradiation followed by TME in 3 of 15, APR (abdominal perineal resection) in 2 of 15 , pelvic exenteration with or without sfincter preservation in 8 of 15 and no operation in 2 of 15 patients. The remaining 20 of 35 patients had a tumor free MRF on MRI. About 15 of these 20 patients received short course radiotherapy $(5 \times 5$ Gy) followed by TME. Histologic evaluation of the surgical specimen showed in all of these 15 patiens a tumor free MRF. The remaining 5 of 20 patients with a predicted tumor free MRF on MRI received long courses of chemoradiation based on adverse prognostic risk factors such as advanced nodal disease and distal location. Three of these 5 patients were treated with TME, 1 of 5 with APR and 1 of 5 received no operation.
Table 1. CT vs. reference standard MRI for the prediction of the tumor relationship to the MRF at patient level.

\begin{tabular}{lcc}
\hline CT observer 1: & Free MRF & ${\text { Invaded } \mathrm{MRF}^{\mathrm{a}}}$ \\
\hline Free & 10 & 2 \\
Threatened & 2 & 1 \\
Invaded & 8 & 12 \\
CT observer 2: & & \\
\hline Free & 7 & 3 \\
Threatened & 2 & - \\
Invaded & 11 & 12 \\
Total: & 20 & 15 \\
\hline
\end{tabular}

Note: Free $\mathrm{MRF}=$ measured distance between tumor and MRF of $\geq 1 \mathrm{~mm}$. Tumor threatened MRF = distance between tumor and $\mathrm{MRF}$ of $<1 \mathrm{~mm}$. Invaded MRF = tumor in contact with MRF

${ }^{a}$ None of the patients had a tumor threatened MRF on MRI

\section{Performance of CT at patient level}

Staging failures of CT for the prediction of tumor invasion of the MRF occurred in 12/35 (34\%) patients for observer 1 and 16/35 (46\%) for observer 2 (Table 1). The majority of these failures were based on the overstaging of the tumor relation to the MRF (obs 1: $10 / 12=83 \%$; obs 2: $13 / 16=81 \%$ ). The area under the ROC curve (AUC), accuracy, sensitivity, specificity, PPV, and NPV are displayed in Table 2. The inter-observer agreement expressed by the linear weighted kappa was 0.34 (95\% CI: $0.04,0.64)$.

\section{Analysis of different anatomical locations}

The results of CT for the evaluation of tumor invasion at different anatomical locations are shown in Table 3. There was a statistically significant difference between the performance of $\mathrm{CT}$ at the mid-high lateral-posterior rectum compared to the low anterior rectum for both observers (obs.1: $\mathrm{AUC}=0.88$ vs. $0.50, P=0.040$; obs 2: $\mathrm{AUC}=0.84$ vs. $0.31, P=0.003$ ). Overstaging occurred in $87 \%(27 / 31)$ of the staging failures of CT for observer $1,83 \%$ (33/40) for observer 2 and were mainly observed at the lower rectum (Figs. 1, 2). CT failed to visualize the rectal tumor at 1 of 4 anatomical locations with tumor invasion of the MRF for observer 1 and 5 of 7 for observer 2 (Fig. 3). Aditionally, observer 1 predicted in 7 of 120 anatomical locations with a normal rectal wall on MRI tumor invasion of the MRF and in 11 of 120 for observer 2 (Fig. 4).

\section{Discussion}

The present study evaluated the accuracy of MDCT for the prediction of tumor invasion of the MRF with MRI as reference standard. We found a poor accuracy (54$66 \%)$, AUC (0.62-0.71) and high inconsistency among observers for the prediction of tumor invasion of the MRF. Evaluation of the staging accuracy of CT at different anatomical locations showed very poor AUCs 
Table 2. Performance of CT for the prediction of tumor invasion of the MRF at patient level.

\begin{tabular}{lll}
\hline & Observer 1 & Observer 2 \\
\hline AUC $(95 \% \mathrm{CI})$ & $0.71(0.54,0.88)$ & $0.62(0.43,0.81)$ \\
Accuracy & $66 \%(23 / 35)$ & $54 \%(19 / 35)$ \\
Sensitivity & $87 \%(13 / 15)$ & $80 \%(12 / 15)$ \\
Specificity & $50 \%(10 / 20)$ & $35 \%(7 / 20)$ \\
PPV & $57 \%(13 / 23)$ & $48 \%(12 / 25)$ \\
NPV & $83 \%(10 / 12)$ & $70 \%(7 / 10)$ \\
\hline
\end{tabular}

Note: $\mathrm{AUC}=$ Area under the receiver operating characteristic curve; $(95 \% \mathrm{CI})=95 \%$ confidence interval; $\mathrm{PPV}=$ positive predictive value; $\mathrm{NPV}=$ negative predictive value

Table 3. Performance of CT for the prediction of tumor invasion of the $\mathrm{MRF}$ at different anatomical locations.

\begin{tabular}{|c|c|c|c|c|}
\hline \multirow[t]{2}{*}{ Locations } & \multicolumn{2}{|c|}{$\begin{array}{l}\text { MRF } \\
\text { invasion }\end{array}$} & \multirow{2}{*}{$\begin{array}{l}\text { Observer } 1 \\
\text { AUC }(95 \% \mathrm{CI})\end{array}$} & \multirow{2}{*}{$\begin{array}{l}\text { Observer } 2 \\
\text { AUC }(95 \% \mathrm{CI})\end{array}$} \\
\hline & - & + & & \\
\hline Low anterior & 3 & 8 & $0.50(0.10,0.90)^{*}$ & $0.31(0.00,0.64)^{*}$ \\
\hline Low lat-post & 32 & 3 & $0.78(0.60,0.97)$ & $0.58(0.19,0.96)$ \\
\hline Mid anterior & 10 & 7 & $0.71(0.46,0.97)$ & $0.66(0.35,0.97)$ \\
\hline Mid-high lat-post & 77 & 8 & $0.88(0.73,1.00)^{*}$ & $0.84(0.66,1.00)^{*}$ \\
\hline All locations** & 122 & 26 & $0.82(0.73,0.92)$ & $0.70(0.56,0.84)$ \\
\hline
\end{tabular}

Note: $\mathrm{AUC}=$ area under the ROC curve with $95 \%$ confidence interval; $-=$ number of cases with a tumor free MRF predicted on MRI; $+=$ number of cases with a tumor threatened or invaded MRF * Statistically significant difference $P \leq 0.04$

** Locations with normal rectal wall on MRI were excluded from analysis $(120 / 268)$

(0.31-0.50) for low-anterior tumors, but the performance significantly improved for mid-high lateral-posterior located rectal tumors (AUC: $0.84-0.88 ; P \leq 0.04$ ).

Some studies on the performance of MDCT for staging of rectal cancer have focused on the prediction of T stage (TNM-staging classification) [13-17]. Matsuoka et al. found in a study of 21 patients comparing MDCT with MRI an agreement of $95 \%$ between MDCT and histology, with no difference between MDCT and MRI. Kulinna et al. reported an accuracy of $86 \%$ in a study of 92 patients [15]. The results of Sinha et al. showed an accuracy of $87 \%$ for the prediction of $\mathrm{T}$ stage in $69 \mathrm{pa}-$ tients [17]. The study of Taylor et al. addressed the clinical important prediction of the tumor relationship to the MRF and reported a poor agreement between MDCT, MRI and histology (kappa 0.06-0.15) in 42 patients treated with a short course of radiotherapy $(5 \times 5$ Gy) or surgery only [13]. The NPV of both MRI and CT was good (82\% and $85 \%$ ), but both modalities had a very low PPV (25\% and 31\%). These results confirm our findings of overstaging with CT, although the overstaging of MRI is rather surprising considering the high accuracy reported in several previous studies [8-11]. The adopted wide cutoff $(<5 \mathrm{~mm})$ for the definitition of tumor threatening and invasion of the MRF might have been responsible for this substantial overstaging [11].
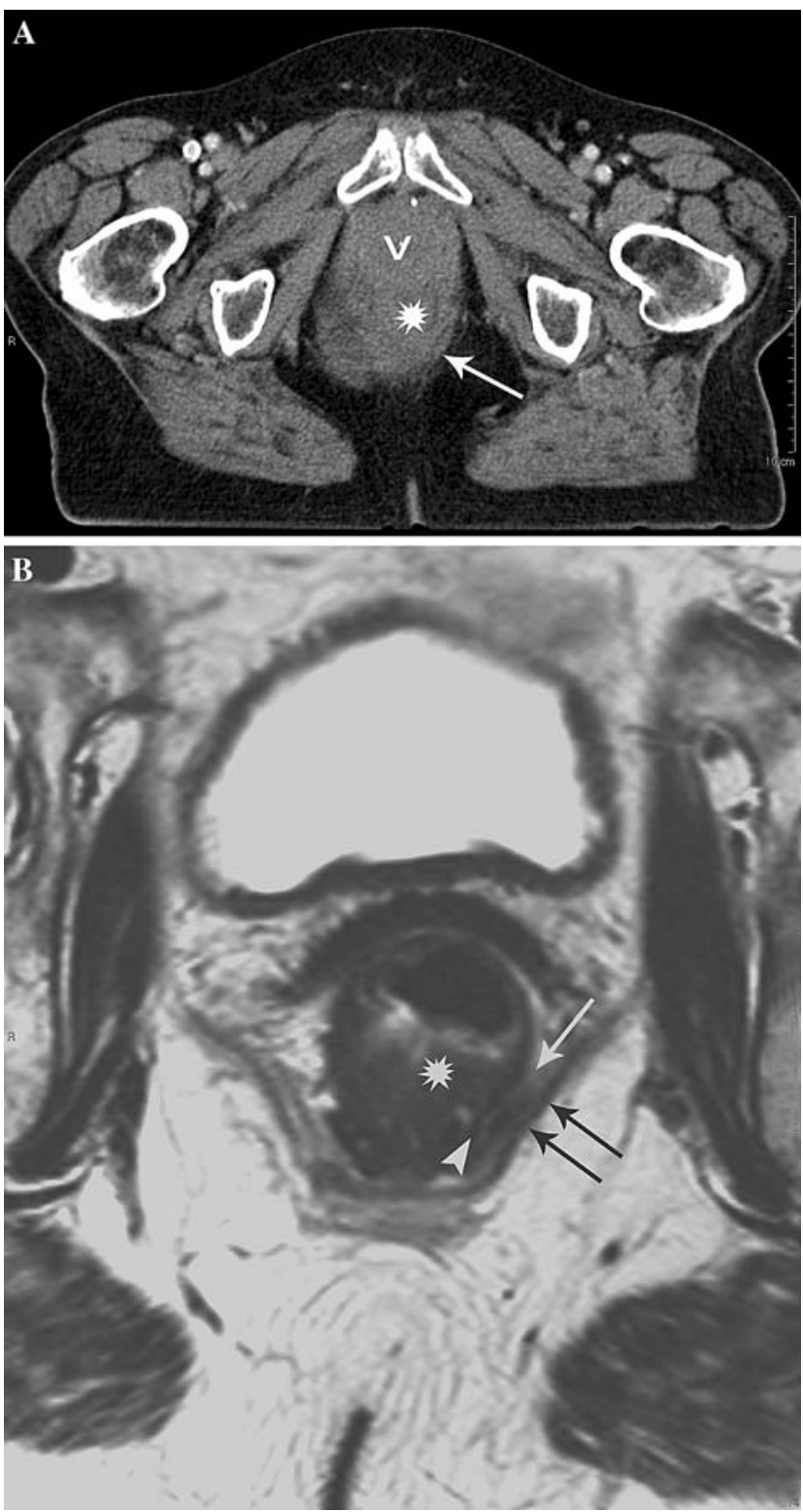

Fig. 1. Poor anatomical detail on CT leading to overestimation of tumor invasion of the MRF in distal rectal tumors. A Axial MS-CT image of distal rectal cancer. The tumor (asterisk) is difficult to delineate and no fat pad can be seen between the tumor and the pelvic floor (arrow) suggesting invasion of the MRF. B Axial T2-weighted MR image at the same level shows a tumor free MRF represented by a partial intact muscular rectal wall layer (arrowhead) and a minimal fat pad (arrow) inbetween the tumor and the pelvic floor muscles (double arrow).

Wolberink et al. report in a retrospective study of 125 with and without a short course of radiotherapy on the value of conventional $\mathrm{CT}$ in predicting MRF invasion [18]. The area under the curve of the ROC curve ranged between 0.697 and 0.813 , and is comparable to the present study. The sensitivity in this study was just below $50 \%$, and the majority of false negatives occurred in tu- 

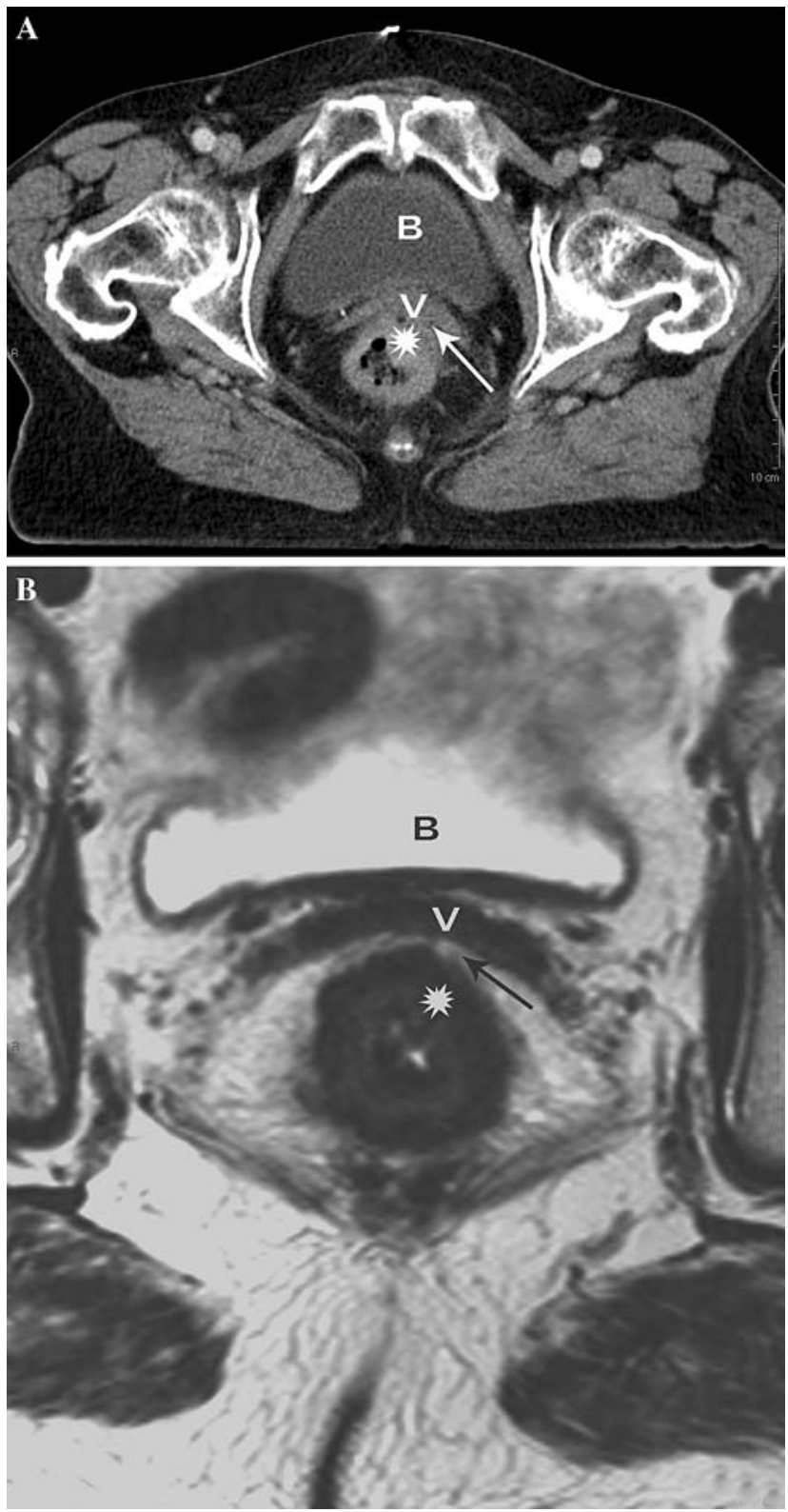

Fig. 2. Another example of poor anatomical detail on CT causing overestimation of MRF invasion in difficult anatomical regions. A Axial MS-CT image of distal rectal cancer (asterisk) showing tumor invasion of the anterior MRF (arrow) and vagina (v). B Axial T2-weighted MR images at the same level shows a tumor free anterior MRF and vagina ( $v$ ) represented by a well-appreciated fat pad (arrow) between the tumor (asterisk) and the anterior MRF.

mors located in the distal anterior rectum. The present study confirms the difficulty in interpreting these tumors even on MDCT.

Our results showed a general overstaging of the tumor relation to the MRF on CT. The main causes were: a poor localization of the tumor in the rectum, leading to misinterpretation of a thickened wall as tumor and vice versa; inability to detect a (partial) intact muscular rectal wall;
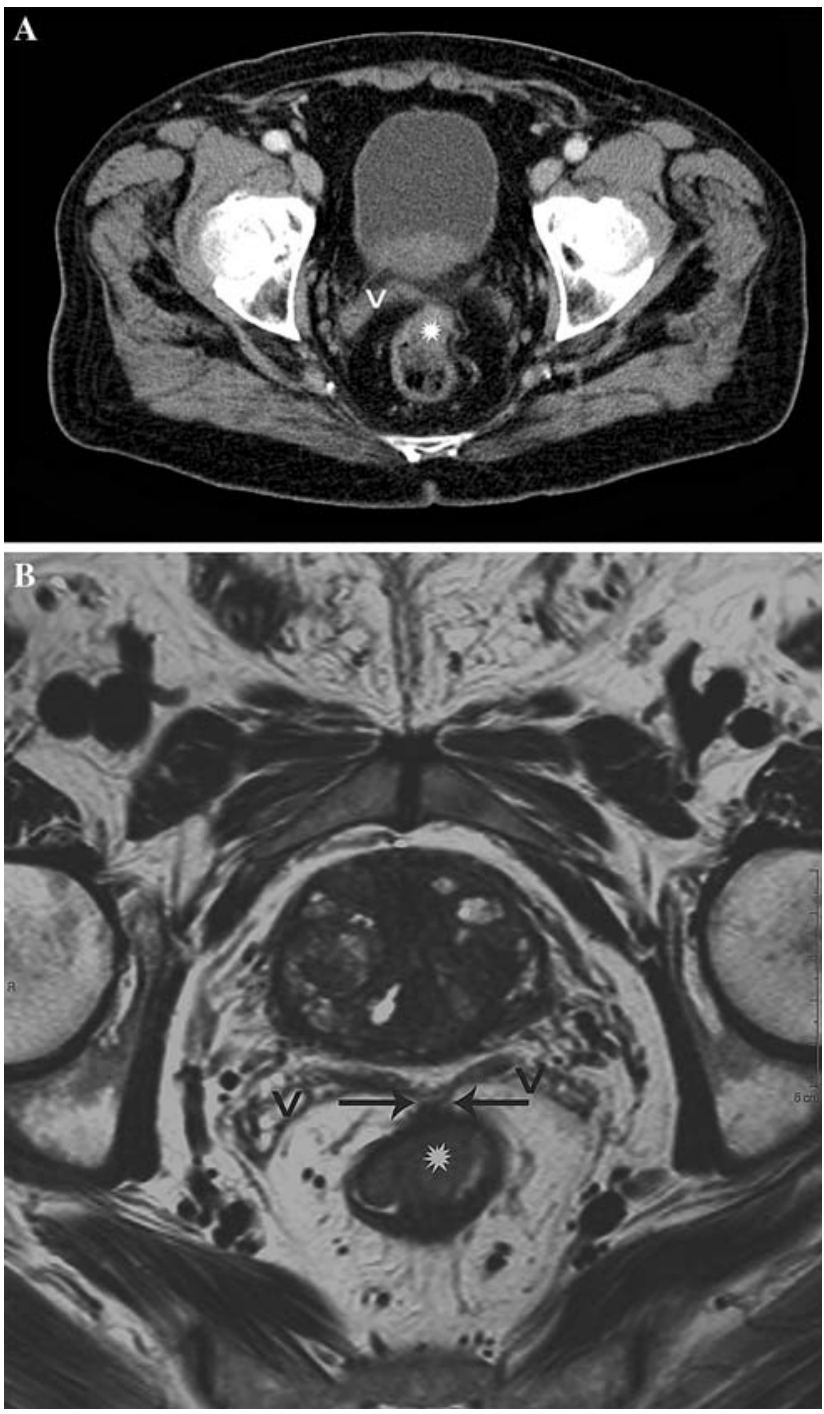

Fig. 3. Difficult visualization of the tumor localization on CT leading to underestimation of tumor invasion of the MRF. A Axial MS-CT image. The rectal tumor located in the anterior rectum (asterisk) at the level of the seminal vesicles (v) is difficult to appreciate due to partial collapse of the rectal lumen and suboptimal soft tissue contrast resolution. B Axial T2-weighted MR image at the same level illustrates an optimal visualization of the tumor in the rectal wall due to high anatomical detail. Also the tumor spread into the anterior MRF (arrows) is well appreciated because of the high soft tissue contrast resolution ( $\mathrm{V}=$ seminal vesicles).

and a poor appreciation of a small fat pad inbetween the tumor and the MRF. Despite major progress of image quality with the Multi-detector row technique, its poor soft tissue contrast resolution compared to MRI remains $[21,22]$. High anatomical detail is essential for the evaluation of distal tumors as we experienced in the majority of our CT staging failures. Tumor penetration of the distal rectal wall almost directly results in tumor invasion of the MRF due to its close relation [23]. This close relation of anatomy restricts the application of rectal inflation by 

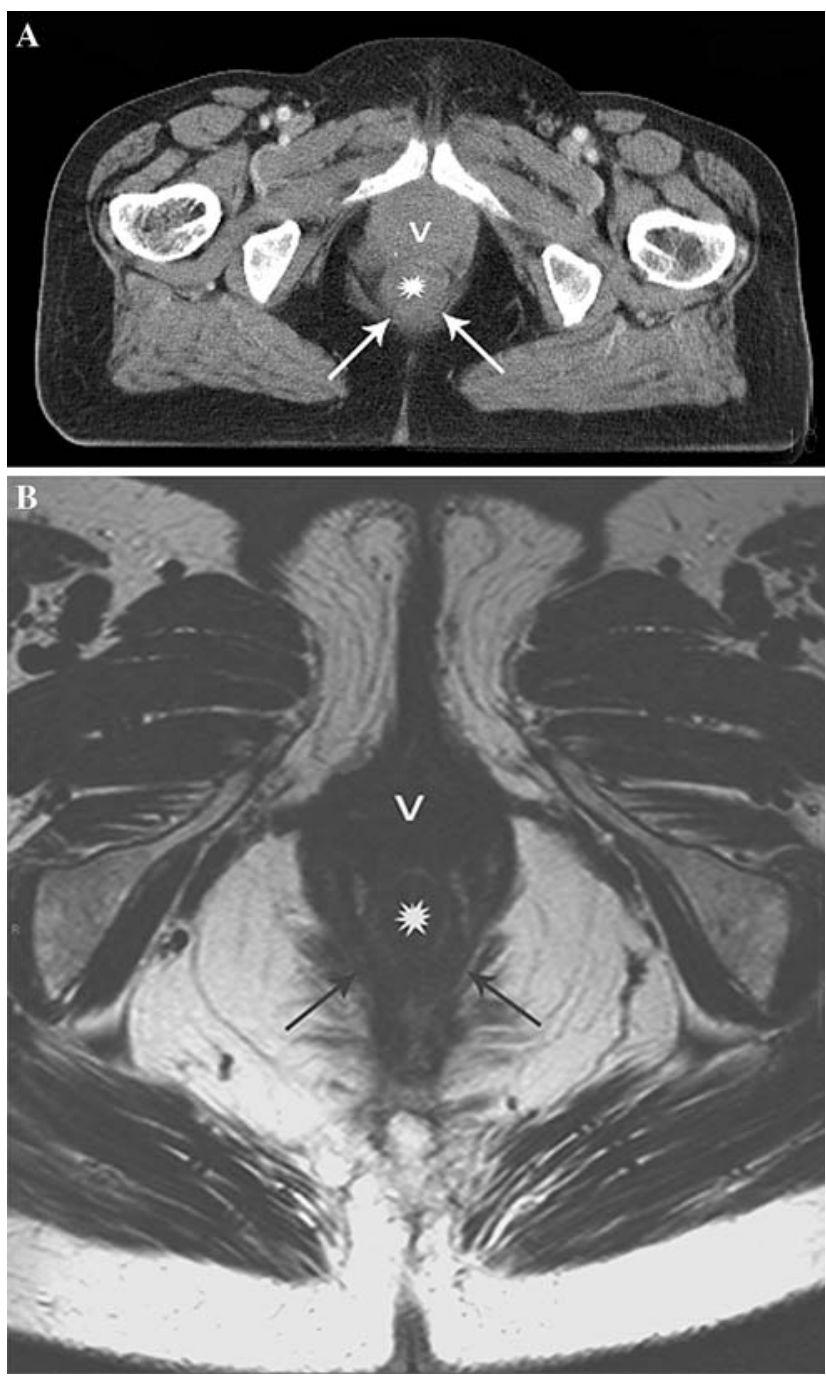

Fig. 4. Normal rectal wall staged as tumor invasion of the MRF on CT due to insufficient anatomical detail. A Axial MSCT image suggest a thickened rectal wall interpreted as distal tumor (asterisk) contacting the pelvic floor (arrows) and vagina (v). B Axial T2-weighted MR image at the same level clearly depicts a normal rectal wall (asterisk) as well as surrounding anatomy (arrows $=$ pelvic floor; $v=$ vagina).

contrast enemas which has been generally used in MDCT studies in order to better appreciate the tumor location [14-16]. The inflation of the rectum causes stretching of the perirectal fat plane and reduces the distance to the MRF leading to overestimation of tumor invasion [24].

The soft tissue contrast resolution appeared to be far less critical in the mid-upper rectum, reflected by the high AUCs (0.84-0.88) for both observers, significantly better than for low-anterior rectal tumors. The presence of a broad non truncated fat manchet surrounding the rectum at these levels gives sufficient contrast between tumor and MRF.

A major point of concern is the poor inter-observer agreement (kappa $=0.34$ ) of CT despite the use of objective criteria for tumor invasion of the MRF. High consistency among observers with different levels of experience is one of the most important pre-requisites for the general acceptance of a staging technique. The consistent results among observers and repeatedly reported high accuracy for the prediction of MRF invasion has contributed to the general acceptance of MRI as the most appropriate selection tool for neo-adjuvant and surgical therapy $[8,9,25]$.

When considering the results of the present study, one must keep in mind that the design has some limitations.We used reconstructed CT slices of $5 \mathrm{~mm}$ which were evaluated in the axial plane only. The use of thinner slices might improve the prediction of MRF invasion in difficult anatomical regions, however, this has never been studied. The only reports on this subject have shown improved $\mathrm{T}$ staging by multi planar reconstruction (MPR) [14, 17]. The main limitation is related to the use of MRI as the reference standard, rather than histology. As argued in the introduction this has become virtually impossible since patients with a tumor that invades or threatens the MRF are currently treated with long courses of chemoradiation. Studying only patients who are treated with surgery only or a short course of preoperatieve radiotherapy creates selection bias of non locally advanced rectal cancer, and excludes those patients who are most at risk for involvement of the MRF. $[13,16]$. Other authors have compared imaging after neoadjuvant chemoradiation with histology, but this is problematic because of the difficulties in interpreting radiofibrosis, and does not give an answer to the question of the relation of the tumor to the MRF before chemoradiation $[9,26]$. MRI is the only modality that has been extensively evaluated for the prediction of tumor invasion of the MRF and several studies have agreed upon its high accuracy [8-11, 25]. Based on the current evidence, we feel that MRI gives the best available representation of the tumor extension to the MRF prior to chemoradiation and therefore could serve as a reference standard.

\section{Conclusion}

Multi-detector row CT has a poor accuracy for the identification invasion of the MRF in low-anterior located tumors. The accuracy of CT significantly improves for tumors located in the mid to high rectum. There is a high inconsistency among readers.

Open Access. This article is distributed under the terms of the Creative Commons Attribution Noncommercial License which permits any noncommercial use, distribution, and reproduction in any medium, provided the original author(s) and source are credited.

\section{References}

1. Heald RJ (1987) Local recurrence of rectal cancer. Dis Colon rectum 30:572 
2. Enker WE (1997) Total mesorectal excision-the new golden standard of surgery for rectal cancer. Ann Med 29:127-133

3. Kapiteijn E, Marijnen CA, Nagtegaal ID, et al. (2001) Preoperative radiotherapy combined with total mesorectal excision for resectable rectal cancer. N Engl J Med 345:638-646

4. Sauer R, Beckers H, Hohenberger W, et al. (2004) Preoperative vs postoperative chemoradiotherapy for rectal cancer. N Engl J Med 351:1731-1740

5. Valentini V, Glimelius B, Minsky BD, et al. (2005) The multidisciplinary rectal cancer treatment: main convergences, controversial aspects and investigational areas which support the need for an European consensus. Radiother Oncol 76:241-250

6. Quirke P, Dixon MF (1988) The prediction of local recurrence in rectal adenocarcinoma by histopathological examination. Int $\mathbf{J}$ Colorectal Dis 3:127-131

7. Nagtegaal ID, Marijnen CA, Kranenbarg E, et al. (2002) Circumferential margin involvement is still an important predictor of local recurrence in rectal carcinoma: not one millimeter but two millimeters is the limit. Am J Surg Pathol 26:350-357

8. Beets-Tan RG, Beets GL, Vliegen RF, et al. (2001) Accuracy of magnetic resonance imaging in prediction of tumor-free resection margin in rectal cancer surgery. Lancet 357:497-504

9. MERCURY study group(2006) Diagnostic accuracy of preoperative magnetic resonance imaging in predicting curative resection of rectal cancer: prospective observational study. BMJ 333:779-790

10. Lahaye MJ, Engelen SM, Nelemans PJ, et al. (2005) Imaging for predicting the risk factors - the circumferential resection margin and nodal disease - of local recurrence in rectal cancer: a metaanalysis. Semin Ultrasound CT MR 26:259-268

11. Brown G, Radcliffe AG, Newcombe RG, et al. (2003) Preoperative assessment of prognostic factors in rectal cancer using high-resolution magnetic resonance imaging. Br J Surg 90:355-364

12. Taylor A, Sheridan M, Mc Gee S, et al. (2005) Pre-operative staging of rectal cancer by MR imaging: results of a UK survey. Clin Radiol 60:579-586

13. Taylor A, Slater A, Mapstone N, et al. (2007) Staging rectal cancer: MRI compared to MDCT. Abdom Imaging 32:323-327

14. Kulinna C, Eibel R, Matzek W, et al. (2004) Staging of rectal cancer: diagnostic potential of multiplanar reconstructions with MDCT. AJR 183:421-427
15. Matsuoka H, Nakamura A, Masaki T, et al. (2003) A prospective comparison between multidetector-row computed tomography and magnetic resonance imaging in the preoperative evaluation of rectal carcinoma. Am J Surg 185:556-559

16. Kulinna C, Scheidler J, Strauss T, et al. (2004) Local staging of rectal cancer: assessment with double-contrast multi-slice computed tomography and transrectal ultrasound. J Comput Assist Tomogr 28:123-130

17. Sinha R, Verma R, Rajesh A, et al. (2006) Diagnostic value of multi-detector row $\mathrm{CT}$ in rectal cancer staging: comparison of multiplanar and axial images with histopathology. Clin Radiol 61:924-931

18. Wolberink S, Beets-Tan RG, de Haas-Kock D, et al. (2007) Conventional CT for the prediction of an involved circumferential resection margin in primary rectal cancer. Dig Dis 25:80-85

19. Hanley J, McNeil B, et al. (1983) A method of comparing the areas under receiver operating characteristics curves derived from the same cases. Radiology 148:839-843

20. Cohen J (1986) Weighted kappa: nominal scale agreement with provision for scaled disagreement or partial credit. Psychol Bull 70:213-230

21. Klingenbeck-Regn K, Schaller S, Flohr T, et al. (1999) Subsecond multi-slice computed tomography: basics and applications. Eur $\mathbf{J}$ Radiol 31:110-124

22. Schima W (2006) MRI of the pancreas: tumors and tumor-simulating processes. Cancer Imaging 6:199-203

23. Salerno G, Sinnatamby C, Branagan G, et al. (2006) Defining the rectum: surgically, radiologically and anatomically. Colorectal Dis 8(Suppl 3):5-9

24. Slater A, Halligan S, Taylor SA, et al. (2006) Distance between the rectal wall and mesorectal fascia measured by MRI: effect of rectal distention and implications for preoperative prediction of a tumorfree circumferential resection margin. Clin Radiol 61:65-70

25. Vliegen RF, Beets GL, von Meyenfeldt M , et al. (2005) Rectal cancer-MR imaging in local staging: is gadolinium-based contrast material helpful?. Radiology 234:179-188

26. Peschaud F, Cuenod CA, Benoist S, et al. (2005) Accuracy of magnetic resonance imaging in rectal cancer depends on location of the tumor. Dis Colon Rectum 48:1603-1609 